\title{
Needs Assessment of Family With Neonates Hospitalized in Neonatal Intensive Care Unit
}

\author{
Sedigheh Razzaghi \\ Babol University of Medical Sciences \\ Yadollah Zahed Pasha \\ Babol University of Medical Sciences \\ Karimollah hajian-Tilaki \\ Babol University of Medical Sciences \\ Sousan valizadeh \\ Tabriz University of Medical Sciences \\ Afsaneh Arzani ( $\sim$ arzaniaf@gmail.com ) \\ Babol University of Medical Sciences
}

\section{Research Article}

Keywords: Neonatal Intensive Care Unit, Neonatal, Hospitalization, Parents, Needs Assessment

Posted Date: March 15th, 2021

DOI: https://doi.org/10.21203/rs.3.rs-288283/v1

License: (c) (i) This work is licensed under a Creative Commons Attribution 4.0 International License. Read Full License 
1 Title of the manuscript: Needs assessment of family with neonates hospitalized in neonatal intensive care unit

\section{Names of all authors:}

3 Sedigheh Razzaghi ${ }^{1}$, Yadollah Zahed Pasha $^{2}$, Karimollah hajian-Tilaki ${ }^{3}$, Sousan valizadeh ${ }^{4}$, Afsaneh arzani $^{5 *}$

4 1-Master of Newborn Nursing, Babol University of Medical Sciences, School of Nursing and Midwifery, Student

5 Research Committee, Babol University of Medical Sciences, Babol, Iran.

6 2-Non-Communicable Pediatric Diseases Research Center,Department of Pediatric, Babol University of Medical

7 Sciences, Babol, I.R. Iran

8 3-Department of Statistics and Epidemiology, School of Medicine, Babol University of Medical Sciences, Babol, I.R.

9 Iran

10 4-Iranian Center for Evidence-Based Practice, Pediatric Nursing Department, School of Nursing and Midwifery,

11 Tabriz University of Medical Sciences, Tabriz, Iran

12 5-Non-Communicable Pediatric Disease Research Center, Health Research Institute, School of Nursing and

13 Midwifery, Babol University of Medical Sciences, Babol, Iran

$14 *$ Corresponding author: Afsaneh Arzani Non-Communicable Pediatric Disease Research Center, Health Research

15 Institute, School of Nursing and Midwifery, Babol University of Medical Sciences, Ganjafrooz Street, Babol,

16 Mazandaran, Iran. Tel:09123768639 Email: arzaniaf@gmail.com

17

18

19 
Abstract:

Back ground:

22 The neonate's birth and subsequent hospitalization cause families to experience various needs. Assessing the family

23 needs can lead identify the real needs of parents, provide qualitative services and implement family-centered care by

24 nurses. The aim of this study was to assess the needs of families with neonates hospitalized in neonatal intensive care

25 unit.

\section{Materials and Methods:}

27 This cross-sectional study was conducted on 360 parents of neonates admitted to NICU in northern Iran in 2018-

28 2019. Data were collected by demographic questionnaire of parents and neonates and NICU Family Needs Inventory

29 (NFNI) of Ward with 5 dimensions of proximity, assurance, information, comfort and support. Data were analyzed 30 using independent t-test, Pearson and Spearman correlation coefficients.

\section{Results:}

32 The most important dimensions of family needs, proximity to infant (93.59\%), information (90.53\%), assurance $33(89.18 \%)$, comfort $(86.70 \%)$ and support $(80.78 \%)$. Comparison indicated that the dimensions of need including 34 assurance of multiparous parents $(\mathrm{P}<0.001)$ and comfort in parents with term infants $(\mathrm{P}<0.02)$ were significantly more 35 than the rest.

36 Conclusion:

37 According to the results of this study, the most important dimension of family needs is proximity to infants. Therefore, 38 it is recommended that the relevant authorities should take effective measures to provide parent-infant proximity. 


\section{Back ground:}

45 Annually, more than $15 \%$ of live neonates worldwide are admitted to the neonatal intensive care unit (NICU)(1). In

46 Iran, infant mortality is estimated at 12 per thousand live births, the main cause of which is premature birth. The birth

47 rate of preterm infants in Iran is $10 \%$, most of whom need to be admitted to the NICU(2). The NICU is a dedicated

48 place to treat and take care of the vulnerable neonates including preterm neonate and newborns with congenital

49 malformations (3). Hospitalization of infants affects the normal routine of life, well-being and family functioning

$50(4,5)$, and parents play a new parental role under adverse conditions such as fear of neonatal death and illness, physical

51 separation from the infant and uncertain future health (6). The NICU environment, equipment, alarms and medical

52 measures provided by health care providers are sources of stress for parents (7). These stressors disrupt the parent-

53 infant relationship physically, mentally and psychologically and cause many emotional problems for parents (8).

54 Hence, identifying family needs reduces stress and emotional problems in parents (9). One of the best and most

55 effective ways to prevent injuries caused by hospitalization is family-centered care by nurses and family involvement

56 in infant care (10). Family-centered care is a caring philosophy that sees the family as the most important and

57 influential member of a baby's health and well-being(11). Family-centered care includes a wide range of educational,

58 psychological, social and behavioral activities of nurses, in all of which family status is at the heart of the care program

59 (12). The first step in implementing the family-centered care is to identify parental needs by nurses and apply those

60 needs in their care program (13) since each parent has unique needs. and nurses' understanding of parental needs is

61 different from the actual needs of parents (14).The results of Hashim et al. suggested that information, assurance and

62 proximity to the infant are the most important needs of parents (15). In 2018, Qi-Yuanlyu et al. showed that the

63 families of hospitalized children with cancer (chronic illness) demanded support, comfort and information (16). In

64 another study, Sargent reported that the most important needs from mothers' perspective were assurance and

65 information (17).

66 Since needs are influenced by culture and there are different cultures in society; therefore, the personality and social

67 characteristics of parents in various regions and cultures may be different (18). There has been limited research on

68 the needs of families in different cultures in Iran so that most studies have focused on the needs of mothers and needs

69 for admission and discharge time(19), but have not addressed the needs of parents(mother and father) at the same

70 time. (14). Parents' needs during neonatal hospitalization have been neglected. As the assessment of needs is essential 
71 in different cultures; hence, the aim of the current study was to assess the needs of families with neonates hospitalized

72 in NICU of some hospitals in northern Iran.

\section{Methods:}

\section{Design and Aim:}

75 The aim of this cross-sectional study was to evaluate the needs of families with neonates hospitalized in NICU.

\section{Sample size:}

77 In the current study, 360 parents with infants admitted to the level III NICU at 4 medical centers as referral centers

78 affiliated to medical universities in northern Iran were selected using convenience sampling method from 2018 to 792019.

80 The method of care in these centers was in the form of newborn individualized developmental care and assessment

81 program (NIDCAP) and kangaroo mother care (KMC); however, there were some challenges related to the lack of

82 unlimited or minimal restrictions on attendance policies. Parents who had no previous experience of infant mortality

83 and hospitalization in the NICU and who had infants with gestational age $>30$ weeks were entered into the present

84 study. Infant mortality and transferring the neonate into another ward were the exclusion criteria for parents.

\section{Measuring Instrument:}

86 Data were collected using the NICU Family Needs Inventory (NFNI) designed by Ward (2001)(20) and demographic

87 questionnaire of parents and neonates. The instrument is an edited version of the Critical Care Family Needs Inventory

88 (CCFNI) designed by Molter (21). The 56-item questionnaire assesses family needs in terms of comfort (getting hope

89 and honest response about infant treatment outcomes), proximity to infants (parental presence at infant's beside),

90 information (information about the neonate's condition), assurance (providing physical and personal comfort to reduce

91 parental stress) and support (getting help from professionals to cope with stress as well as getting emotional and social

92 support from others). Out of 56 questions including infant proximity (8item), assurance(12item), support(18item),

93 information(11item) and comfort (7item ). The questions of questionnaire are scored on a 4-point Likert scale ranging

94 from 1 to 4 (not important, slightly important, important, and very important, respectively). The importance of needs 
was determined based on the parents' response to the items in the questionnaire. The internal reliability coefficient of the questionnaire was 0.92 based on Cronbach's alpha.

\section{Data Collection:}

98 Due to the possibility of the mother's absence in the first days after delivery, the questionnaire was provided to parents

99 from the third day to 24 hours before the baby's discharge. The self-report questionnaire was filled out simultaneously

100 by both parents. Parents had the opportunity to complete the questionnaire and return it to the researcher up to 24

101 hours before discharge. In order to do Ethical consideration, this study followed the guidelines set by Declaration of

102 Helsinki and received ethical approval for human subject by the Ethics Commit-tees of Babol University of Medical

103 Sciences (IR MUBABOL.HRI.REC.1397.020) and written informed consent was obtained from all parents following

104 a detailed description of the purpose of the study

\section{Data Analysis:}

106 After collecting the final data, SPSS 23 was used to analyze the data. Statistical test of Pearson and Spearman 107 correlation coefficients was used to investigate the relationship between family dimensions with some quantitative 108 and qualitative variables. In order to compare the dimensions of the needs of each group with those of another group, 109 the independent statistical t-test was applied, and $\mathrm{p}<0.05$ was considered as significant level. For the dimensions of 110 family needs in questionnaire had different scores, standardization was done to determine the dimensions of family 111 needs based on importance from parents' point of view and the dimensions were calculated based on one hundred.

\section{Result:}

113 In this study, 360 parents were entered into the study. Most of the participating mothers had non-academic education $114(50.2 \%)$ and were housewives $(90.3 \%)$ as well as the majority of participating fathers had non-academic education 115 (53\%) and were self-employed (80.9\%). Most of the newborns were term (51.9\%), boy (54.4\%), first child (50.8\%) 116 and born by cesarean section (71.1\%). Totally (36\%) of infants were hospitalized for respiratory distress syndrome, 117 (19\%) for prematurity, (14\%) for icterus and (31\%) for other illnesses.

118 Comparison of the dimensions of needs according to the parents' importance suggested that proximity to infant, 119 information, assurance, comfort and support were (93.59\%), (90.53\%), (89.18\%), (86.705) and (80.78\%), 120 respectively. 
121 The results of comparing dimensions of family needs with some variables of parents and infants represented that the

122 mean assurance was higher in multiparous parents $(90.27 \pm 6.08)$ than primiparous parents $(88.12 \pm 5.38)$ and the mean

123 comfort was higher in parents of term infants $(88.03 \pm 11.03)$ than those of preterm infants $(85.30 \pm 11.53)$ so that this

124 difference was statistically significant $(\mathrm{p}<0.05)$. There was no significant difference between mothers' needs with

125 education and job ( $>0.05)$ (tables 1,2$)$.

126 Table 1: Comparison of different needs of families with infants hospitalized in NICU based on gestational age,

127 multiparous and primiparous

\begin{tabular}{|c|c|c|c|c|c|c|}
\hline \multirow[t]{3}{*}{ Groups } & Primipara & Multipara & \multirow{2}{*}{\multicolumn{2}{|c|}{ T-test result }} & Term & \multirow{3}{*}{ T-test result } \\
\hline & & & & & & \\
\hline & Mean \pm SD & Mean \pm SD & & Mean \pm SD & Mean \pm SD & \\
\hline \multicolumn{7}{|l|}{ Needs } \\
\hline \multicolumn{7}{|l|}{ dimensions } \\
\hline & & & $\mathrm{t}=1.52 \quad \mathrm{df}=346$ & & & $\mathrm{t}=0.146 \quad \mathrm{df}=358$ \\
\hline \multirow[t]{3}{*}{ Proximity } & $94 \pm 6.49$ & $93.5 \pm 7.56$ & & $93.64 \pm 6.64$ & $93.53 \pm 7.40$ & \\
\hline & & & $\mathrm{p}$-value $=0.25$ & & & $\mathrm{p}$-value $=0.884$ \\
\hline & & & $t=0.687 \quad d f=344$ & & & $\mathrm{t}=-0.849 \quad \mathrm{df}=358$ \\
\hline \multirow[t]{3}{*}{ Information } & $90.83 \pm 7.81$ & $90.22 \pm 9.20$ & & $90.13 \pm 8.56$ & $90.89 \pm 8.46$ & \\
\hline & & & $\mathrm{p}$-value $=0.492$ & & & $\mathrm{p}$-value $=0.396$ \\
\hline & & & $\mathrm{t}=-3.358 \quad \mathrm{df}=358$ & & & $\mathrm{t}=-0.390 \quad \mathrm{df}=358$ \\
\hline \multirow[t]{3}{*}{ Assurance } & $88.12 \pm 5.38$ & $90.27 \pm 6.08$ & & $89.05 \pm 5.87$ & $89.29 \pm 5.80$ & \\
\hline & & & $\mathrm{p}$-value $=<0.001$ & & & $\mathrm{p}$-value $=0.697$ \\
\hline & & & $\mathrm{t}=0.699 \quad \mathrm{df}=358$ & & & $\mathrm{t}=-2.291$ \\
\hline \multirow[t]{3}{*}{ Comfort } & $87.14 \pm 10.67$ & $86.30 \pm 12.03$ & & $85.30 \pm 11.53$ & $88.03 \pm 11.03$ & \\
\hline & & & $\mathrm{p}$-value $=0.485$ & & & $\mathrm{p}$-value $=0.023$ \\
\hline & & & $\mathrm{t}=-0.913 \quad \mathrm{df}=358$ & & & $\mathrm{t}=-1.168 \quad \mathrm{df}=358$ \\
\hline \multirow[t]{2}{*}{ Support } & $80.24 \pm 10.85$ & $81.34 \pm 11.99$ & & $80.04 \pm 10.94$ & $81.45 \pm 11.84$ & \\
\hline & & & $\mathrm{p}$-value $=0.362$ & & & $\mathrm{p}$-value $=0.243$ \\
\hline
\end{tabular}




\begin{tabular}{llllll}
\hline & Academic & Non-academic & & & \\
& education & education & & Housewife & Employee \\
& & T-test result & & T-test result \\
Groups & Mean \pm SD & Mean \pm SD & & Mean \pm SD & Mean \pm SD
\end{tabular}

Needs dimensions

\begin{tabular}{|c|c|c|c|c|c|c|}
\hline \multirow{3}{*}{ Proximity } & \multirow{3}{*}{$93.45 \pm 6.97$} & \multirow{3}{*}{$93.71 \pm 7.11$} & $\mathrm{t}=-0.353 \quad \mathrm{df}=358$ & & & \multirow[t]{2}{*}{$\mathrm{t}=-0.641 \quad \mathrm{df}=358$} \\
\hline & & & & $93.52 \pm 7.16$ & $94.10 \pm 5.85$ & \\
\hline & & & \multicolumn{3}{|l|}{$\mathrm{p}$-value $=0.724$} & $\mathrm{p}$-value $=0.645$ \\
\hline & & & $\mathrm{t}=-0.511 \quad \mathrm{df}=358$ & & & $\mathrm{t}=0.055$ \\
\hline \multirow[t]{3}{*}{ Information } & $90.29 \pm 8.99$ & $90.75 \pm 8.02$ & & $90.53 \pm 8.66$ & $90.45 \pm 7.11$ & \\
\hline & & & \multicolumn{3}{|l|}{$\mathrm{p}$-value $=0.61$} & $\mathrm{p}$-value $=0.956$ \\
\hline & & & $\mathrm{t}=-0.271 \quad \mathrm{df}=358$ & & & $\mathrm{t}=0.012$ \\
\hline \multirow[t]{3}{*}{ Assurance } & $89.09 \pm 5.93$ & $82.26 \pm 5.73$ & & $89.17 \pm 5.96$ & $89.16 \pm 4.48$ & \\
\hline & & & \multicolumn{3}{|l|}{$\mathrm{p}$-value $=0.787$} & $\mathrm{p}$-value $=0.99$ \\
\hline & & & $\mathrm{t}=0.554$ & & & $\mathrm{t}=-1.22$ \\
\hline \multirow[t]{3}{*}{ Comfort } & $87.05 \pm 11.55$ & $86.39 \pm 11.15$ & & $86.55 \pm 11.66$ & $88.33 \pm 7.74$ & \\
\hline & & & \multicolumn{3}{|l|}{$\mathrm{p}$-value $=0.58$} & p-value $=0.228$ \\
\hline & & & $\mathrm{t}=-0.469 \quad \mathrm{df}=358$ & & & $\mathrm{t}=-0.284$ \\
\hline \multirow[t]{2}{*}{ Support } & $80.49 \pm 12.11$ & $81.06 \pm 10.72$ & & $80.72 \pm 11.61$ & $81.3 \pm 9.54$ & \\
\hline & & & $\mathrm{p}$-value $=0.64$ & & & $\mathrm{p}$-value $=0.776$ \\
\hline
\end{tabular}

130 Relationship between dimensions of parental need and some qualitative and quantitative variables demonstrated that

131 there was a significant direct relationship between assurance with paternal and maternal ages, number of childbirths

132 and birth order so that the need for assurance was enhanced with increasing age of parents, number of births and birth

133 order. Moreover, there was also a significant inverse relationship between paternal education and assurance as well as

134 between maternal education and proximity. The needs for proximity and assurance were lower in parents with

135 increasing maternal and paternal education, respectively (Table 3).

136 Table 3: Correlation between needs dimensions of families with infants hospitalized in NICU and some 137 quantitative and qualitative variables

\begin{tabular}{|c|c|c|c|c|c|c|}
\hline variables & Paternal age & Maternal age & $\begin{array}{l}\text { Numberof } \\
\text { childbirths }\end{array}$ & Birth order & Paternal education & Maternal education \\
\hline & $\mathrm{p}$ & $\mathrm{r}$ & $\mathrm{p}$ & $\mathrm{p}$ & $\mathrm{p}$ & $\mathrm{r}$ \\
\hline
\end{tabular}




\begin{tabular}{|c|c|c|c|c|c|c|c|c|c|c|c|c|}
\hline $\begin{array}{c}\text { Dimensions } \\
\text { needs as }\end{array}$ & & & & & & & & & & & & \\
\hline Assurance & 0.160 & 0.002 & 0.139 & 0.008 & 0.218 & $<0.01$ & 0.242 & $<0.01$ & -0.129 & 0.015 & -0.061 & 0.249 \\
\hline Proximity & -0.015 & 0.779 & -0.029 & 0.588 & -0.061 & 0.249 & -0.047 & 0.375 & -0.090 & 0.087 & -0.063 & 0.023 \\
\hline Information & 0.046 & 0.379 & -0.003 & 0.958 & -0.019 & 0.714 & 0.002 & 0.937 & -0.070 & 0.184 & -0.024 & 0.652 \\
\hline Comfort & -0.034 & 0.514 & -0.001 & 0.982 & -0.015 & 0.776 & -0.012 & 0.825 & 0.053 & 0.316 & 0.008 & 0.886 \\
\hline Support & 0.018 & 0.729 & 0.043 & 0.413 & 0.036 & 0.490 & 0.042 & 0.431 & -0.067 & 0.205 & -0.038 & 0.474 \\
\hline
\end{tabular}

\section{Discussion:}

140 The results of the present study indicated that the needs for proximity to the infant and assurance were more important

141 and the need for support was less important than the rest among families. Like the current study, Liwang et al. found

142 that the need for infant proximity had a higher priority than other dimensions of needs among parents (22). Consistent

143 with the results of the current study, Aemmi et al. concluded that the needs for proximity to infants and assurance

144 were more important among mothers (23). Cleveland et al. acknowledged that nurses should have a positive attitude

145 toward mothers who are able to take care of their infants with their supervision, which helps bring the mother and

146 baby closer together (24). Moreover, Guillaume et al. have demonstrated that the caring attitude of nurses and their

147 regular communication according to the parents' needs are essential prerequisite for parent-infant bonding and greater

148 parental involvement in the NICU (25).

149 Other studies like the ongoing study have reported that the least important need is support $(14,17,20,22)$. The reason 150 may be that health care providers are adequately meeting the support need, or the importance of other needs may be 151 such that the support need is less important than other needs.

152 However, Alsaiari et al exhibited that the most important need from parents' perspective was assurance, proximity and 153 information, which is different from the present results (26). This difference might be due to changes in the 154 questionnaire used in the above studies, which determined the parental needs. Since it was possible for the participated 155 parents to have unlimited visits to the NICU in mentioned studies. Vaskelyte et al. stated that the needs of parents 
based on importance were assurance, proximity to infants, information, support and comfort (27), which is inconsistent with the results of the present study. The assurance need was more felt in parents because the questionnaires of needs assessment in the abovementioned study were completed in the first days of infant hospitalization, parents in the first days needed to feel more assurance and confident about their infant's future and mothers were unable to provide basic care for their infants in the first days due to high anxiety levels. Whereas in the present study, questionnaires were filled out at different times of infant hospitalization, which may affect the type of parental need. In another study, Sargent found that the priority of needs from the mothers' perspective was assurance, information, infant proximity, comfort and support, respectively, which is contrary to the present study (17). In the study of Sargent, only the needs of mothers with neonates admitted to the level III NICU were investigated, while the needs of both mothers and fathers were evaluated in our study and samples were collected from all levels of NICU. Mirjalili et al. expressed that the assurance need was the most important one from mothers' perspective, which is not consistent with the ongoing study (19). This difference may be related to the used questionnaire to determine the maternal need or the time for completing the questionnaire (admission time). In the present study, the need for comfort was more felt in parents with term infants. Multiparous parents reported more need for assurance than primiparous parents. There was no significant difference between mothers' needs and academic/non-academic education. In this way, Alves et al. (2016) illustrated

171 that parents with lower level of education had more needs for comfort and support and with higher level of education

172 had more needs for assurance and comfort (13). In Mundy et al.'s study, no significant difference was found among 173 the dimensions of needs in parents under diploma. Parents with diploma stated more need for support and information, 174 and with academic education reported a greater need for support. There was no significant difference between the 175 needs of primiparous and multiparous parents (14). Mirjalili et al. acknowledged that the need for information was 176 less among mothers with preterm infants than other mothers (19), which is incompatible with the results of the present 177 study. Perhaps, the reason might be that the performed studies were related to the needs at the time of infant, while 178 the current study evaluated the parental need during hospitalization with large sample size. Besides the used 179 questionnaires of both studies were different.

\section{Limitations:}

181 One of the limitations of our study was the inaccessibility to all the cities of northern Iran province since culture, 182 customs and beliefs can influence the type of needs. Therefore, considering the cultural and ethnic diversity, it is 
183 recommended to conduct further studies with different populations culturally and socially in order to identify the needs

184 of various cultures.

185 Conclusion:

186 The present study indicated that the needs for infant proximity and assurance were more important and the need for

187 support was less than the rest among families. Therefore, it is recommended that the results of this study should be 188 considered by maternal and neonatal health policy makers for providing unlimited or least limited possibility to attend 189 in the NICU for families in addition to facilitating the emotional bond between infant and parents in order to enable 190 the stress experienced by the families during the infant hospitalization. Furthermore, identifying and meeting the real 191 needs of the family can be used as a strategy to improve the provided health care and promote family compatibility 192 during infant hospitalization.

\section{List of abbreviation:}

194 NICU: neonatal intensive care unit.

195 NFNI: NICU Family Needs Inventory

196 NIDCAP: newborn individualized developmental care and assessment program

197 KMC: kangaroo mother care

198 CCFNI: Critical Care Family Needs Inventory

$199 \mathrm{p}=\mathrm{P}-$ Value

200 SD=Standard Deviation

201 DF=Degree of freedom

202 Declaration:

203 Ethics approval and consent to participate:

204 In order to do Ethical consideration, this study followed the guidelines set by Declaration of Helsinki and received 205 ethical approval for human subject by the Ethics Commit-tees of Babol University of Medical Sciences (IR 
207 description of the purpose of the study

208 Consent for publication:

209 Not applicable

210 Availability of data and materials:

211 The datasets used and/or analyzed during the current study are available from the first author on reasonable request.

\section{Competing interest:}

213 The authors declare that they have no competing interests.

\section{Funding:}

215 There is no funding support.

\section{Authors contribution:}

217 S.R designed the study, collected and analyzed the data, and wrote the article. Y.Z and S.V reviewed the article K.H 218 analyzed data and wrote article. A.A designed the study and she was a major contributor in writing the manuscript. 219 All authors read and approved the final manuscript.

\section{Acknowledgments:}

221 The authors would like to appreciate the study parents who complete the questionnaires carefully despite their stress

222 and concerns, Babol University of Medical Sciences and Mazandaran University of Medical Sciences for its 223 assistance. This article is derived from a master's thesis in neonatal intensive care.

\section{References:}

225 1. Jones L, Woodhouse D, Rowe J. Effective nurse parent communication: a study of parents' perceptions in the NICU 226 environment. Patient Educ Couns. 2007;69(1-3):206-12.

227 2. Dezvaree N, Alaeekarahroudi F, KhanaliAgan L, TalebiGhane E. The mother-newborn s attachment and its related 228 factors in mothers of hospitalized preterm neonates. J Heal Care. 2016;17(4):340-9.

229 3. Ichijima E, Kirk R, Hornblow A. Parental support in neonatal intensive care units: a cross-cultural comparison 230 between New Zealand and Japan. J Pediatr Nurs. 2011;26(3):206-15.

231 4. Lasiuk GC, Comeau T, Newburn-Cook C. Unexpected: an interpretive description of parental traumas' associated 232 with preterm birth. BMC Pregnancy Childbirth. 2013;13(1):S13. 
233 5. Rich-Edwards JW, Fraser A, Lawlor DA, Catov JM. Pregnancy characteristics and women's future cardiovascular 234 health: an underused opportunity to improve women's health? Epidemiol Rev. 2014;36(1):57-70.

235 6. Araújo AC, Costa CI, others. Social representation of fathers regarding their premature child in the Neonatal 236 Intensive Care Unit. Rev Bras Enferm. 2009;62(5):734-8.

237 7. Kleberg A, Hellström-Westas L, Widström A-M. Mothers’ perception of Newborn Individualized Developmental 238 Care and Assessment Program (NIDCAP) as compared to conventional care. Early Hum Dev. 2007;83(6):403-11.

239 8. Özdemir FK, Alemdar DK. Supporting of the fathers to visit their infants in neonatal intensive care unit decreases 240 their stress level: a pretest--posttest quasi-experimental study. Community Ment Health J. 2017;53(4):490-5.

241 9. Jubinville J, Newburn-Cook C, Hegadoren K, Lacaze-Masmonteil T. Symptoms of acute stress disorder in mothers 242 of premature infants. Adv Neonatal Care. 2012;12(4):246-53.

243 10. Ghasemi M, Dehdari T, Mohagheghi P, Gohari MR. The effect of educational intervention based on theory of 244 planned behavior (TPB) for improving method of care of premature infant by mother. Razi J Med Sci. $245 \quad 2014 ; 20(115): 39-48$.

246 11. Staniszewska S, Brett J, Redshaw M, Hamilton K, Newburn M, Jones N, Taylor L. The POPPY study: developing 247 a model of family-centred care for neonatal units. Worldviews on Evidence-Based Nursing. 2012 Nov;9(4):243-55.

248 12. Heinemann A-B, Hellström-Westas L, Hedberg Nyqvist K. Factors affecting parents' presence with their 249 extremely preterm infants in a neonatal intensive care room. Acta Paediatr. 2013;102(7):695-702.

250 13. Alves E, Severo M, Amorim M, Grande C, Silva S. A short form of the neonatal intensive care unit family needs 251 inventory. J Pediatr (Rio J). 2016;92(1):73-80.

252 14. Mundy CA. Assessment of family needs in neonatal intensive care units. Am J Crit Care. 2010;19(2):156-63.

253 15. Hashim F, Hussin R. Family needs of patient admitted to intensive care unit in a public hospital. Procedia-Social 254 Behav Sci. 2012;36:103-11. 
256 childhood hospitalization for cancer treatment in Mainland China: A qualitative study. J Pediatr Nurs. 2019;44:e66-$257 \mathrm{e} 71$.

258 17. Sargent AN. Predictors of needs in mothers with infants in the neonatal intensive care unit. J Reprod Infant 259 Psychol. 2009;27(2):195-205.

260 18. Sikorova L, Kucova J. The needs of mothers to newborns hospitalised in intensive care units. Biomed Pap Med 261 Fac Univ Palacky Olomouc Czech Repub. 2012 Dec 12;156(4):330-6.

262 19. Mirjalili M, Sabzevari S, Haghdost A, others. Assess the admission needs of the mothers of hospitalized in the 263 neonatal intensive care unit. J Pediatr Nurs. 2015;2(2):1-9.

264 20. Ward K. Perceived needs of parents of critically ill infants in a neonatal intensive care unit (NICU). Pediatr Nurs. $265 \quad 2001 ; 27(3): 281$.

266 21. Molter NC. Needs of relatives of critically ill patients: a descriptive study. Hear lung. 1979;8(2):332-9.

267 22. Wang L, He JL, Fei SL. Perceived needs of parents of premature infants in NICU. West J Nurs Res. $268 \quad 2018 ; 40(5): 688-700$.

269 23. Aemmi SZ, Ahmadi Z, Reyhani T, Haghani H. Comparison of perceptions of nurses and premature infants' 270 mothers about mothers' needs in neonatal intensive care unit. J hayat. 2013;19(2):14-26.

271 24. Cleveland LM. Parenting in the neonatal intensive care unit. J Obstet Gynecol Neonatal Nurs. 2008;37(6):66627291.

273 25. Guillaume S, Michelin N, Amrani E, Benier B, Durrmeyer X, Lescure S, et al. Parents' expectations of staff in the 274 early bonding process with their premature babies in the intensive care setting: a qualitative multicenter study with 60 275 parents. BMC Pediatr. 2013;13(1):18.

276 26. Alsaiari EM, Magarey J, Rasmussen P. An Investigation of the Needs of Saudi Parents of Preterm Infants in the 277 Neonatal Intensive Care Unit. Cureus. 2019;11(1). 

parents' and nurses' perceptions. Medicina (B Aires). 2010;46:43-53.

280 